Supporting Information for

\title{
Living Polymer Chains with Predictable Molecular Weight and Dispersity via Carbanionic Polymerization in Continuous Flow: Mixing Rate as a Key Parameter
}

Jan Morsbach, Axel H. E. Müller, Elena Berger-Nicoletti, Holger Frey*

Institute of Organic Chemistry, Johannes Gutenberg-University (JGU), Duesbergweg 10-14, D-55099 Mainz, Germany 


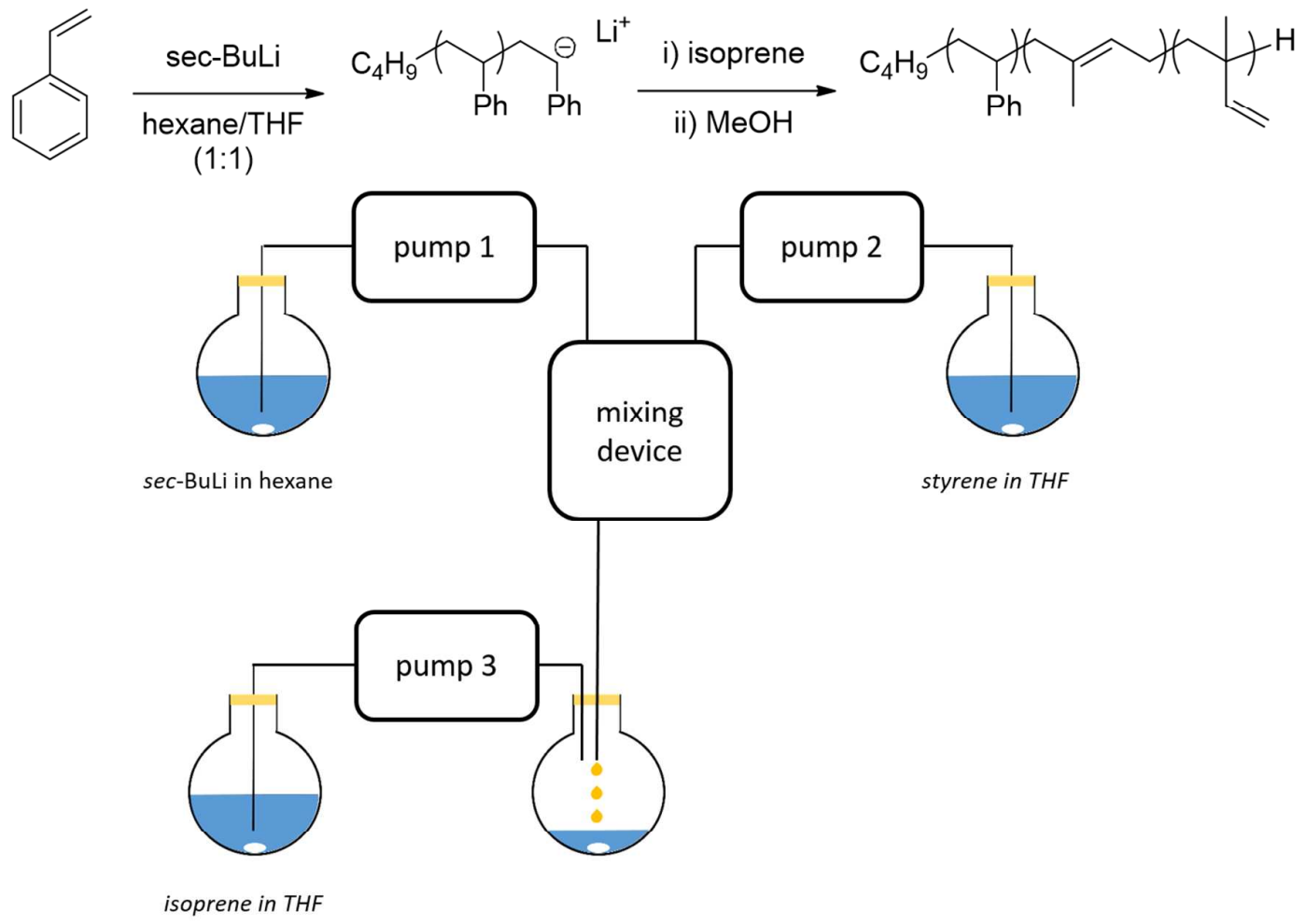

Scheme S1. Reaction scheme (top) and micro reactor setup (bottom) for the direct synthesis of PS- $b$ PI block copolymers via living anionic polymerization.

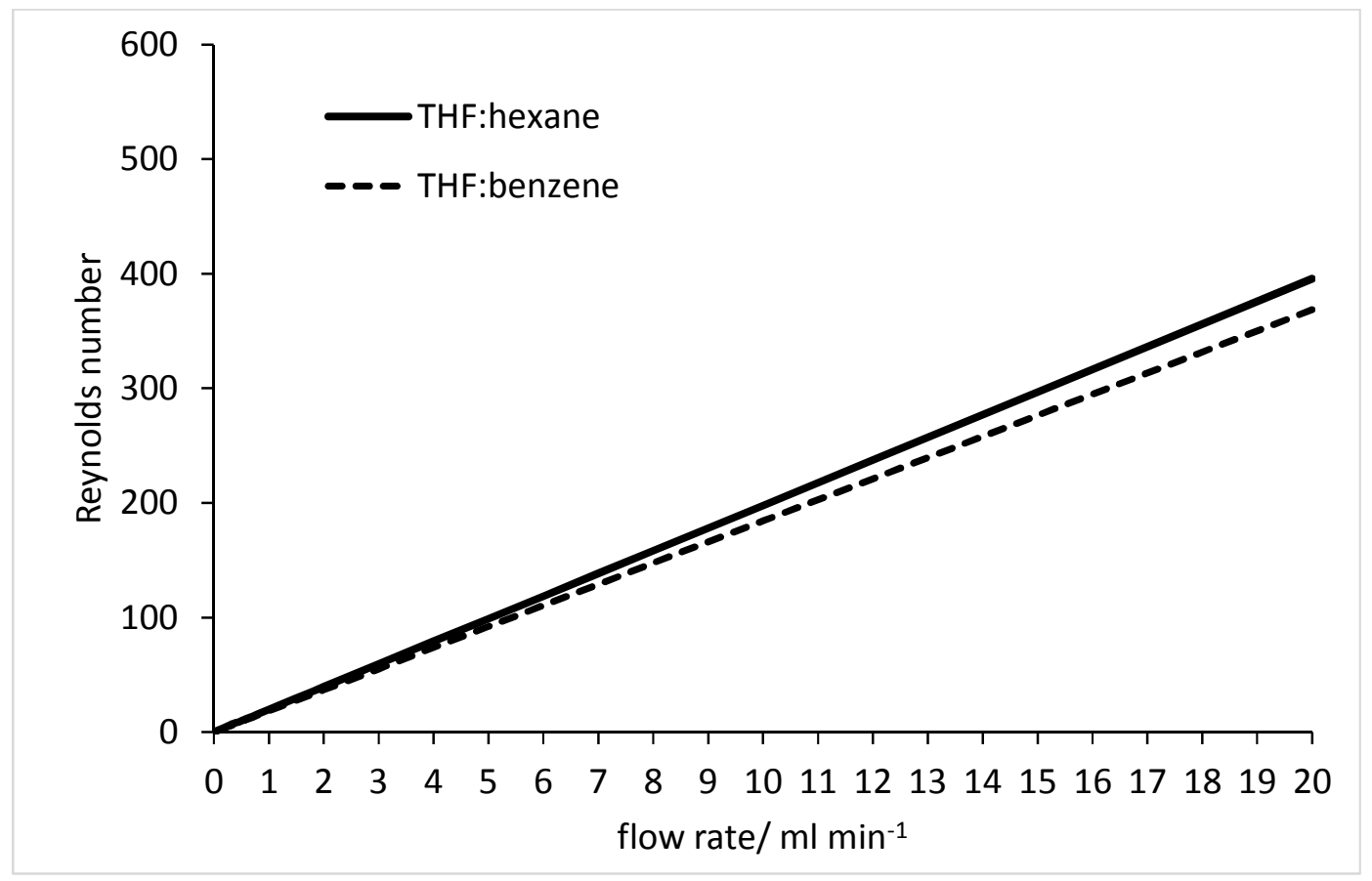

Figure S1: Reynolds number for both solvent mixtures (straight line: THF:hexane for PS, PpMeS; dotted line: THF:benzene for P2VP) over the total flow rate for the steel capillary used. 
a)
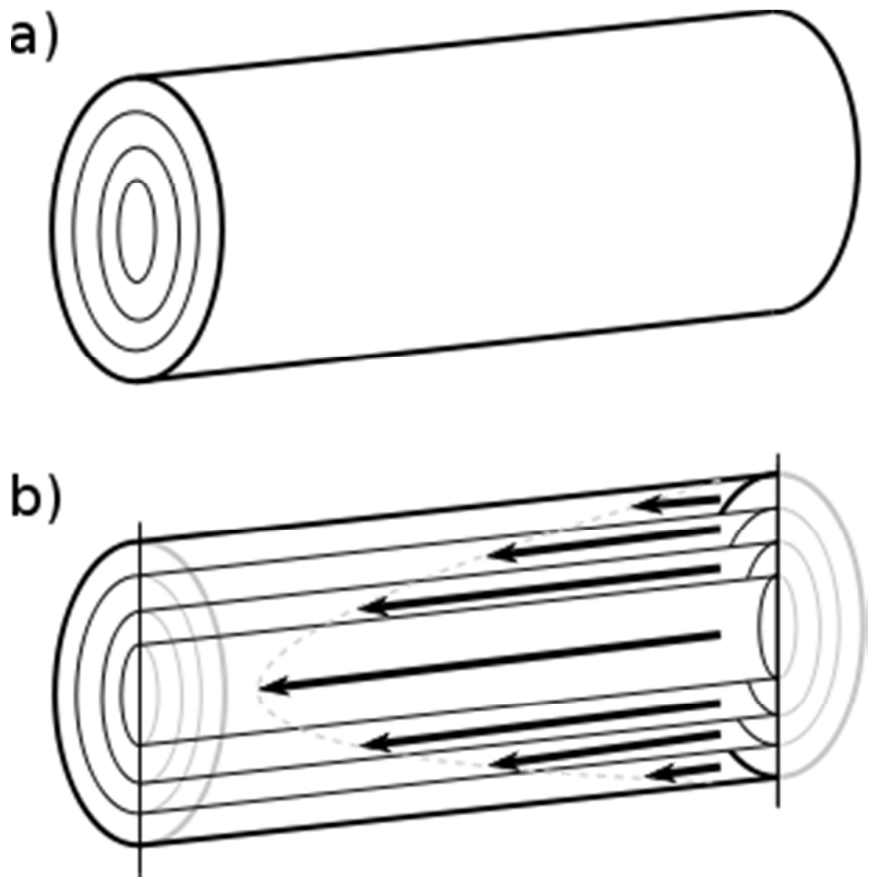

Figure S2: Flow profile in a pipe following the Hagen-Poisseuille law.

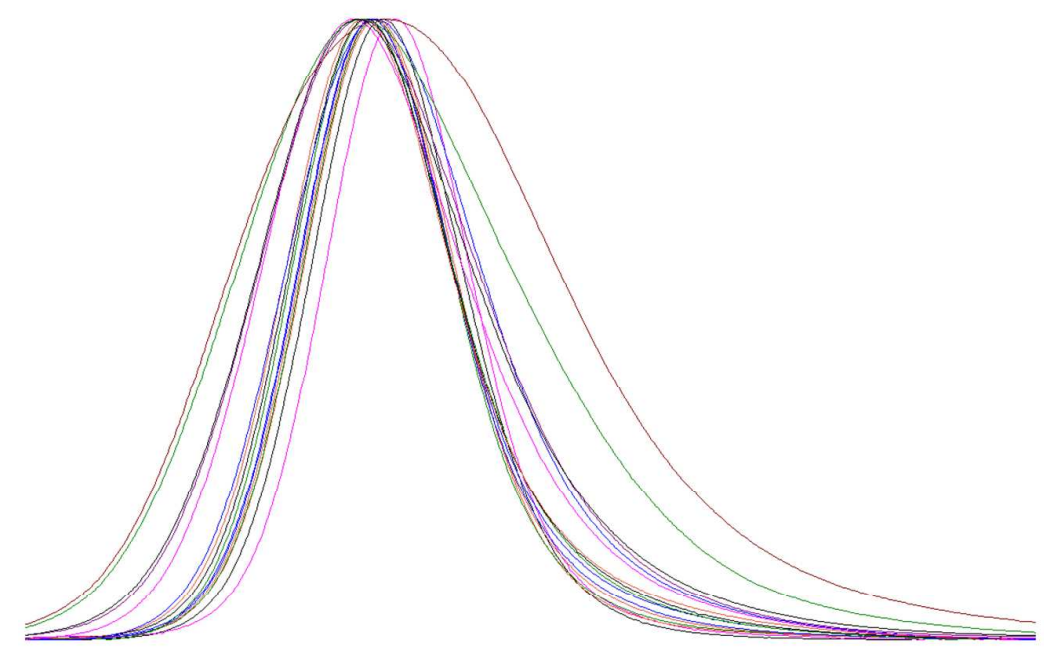

$8.0 \mathrm{ml} \mathrm{min}^{-1} ; \mathrm{PDI}=1.15$

$7.0 \mathrm{ml} \mathrm{min}^{-1} ; \mathrm{PDI}=1.22$

$6.2 \mathrm{ml} \mathrm{min}^{-1} ; \mathrm{PDI}=1.22$

$5.4 \mathrm{ml} \mathrm{min}^{-1} ; \mathrm{PDI}=1.22$

$4.8 \mathrm{ml} \mathrm{min}^{-1}$; $\mathrm{PDI}=1.26$

$4.2 \mathrm{ml} \mathrm{min}^{-1} ; \mathrm{PDI}=1.29$

$-3.6 \mathrm{ml} \mathrm{min}^{-1} ; \mathrm{PDI}=1.28$

$3.2 \mathrm{ml} \mathrm{min}^{-1} ; \mathrm{PDI}=1.34$

$-2.8 \mathrm{ml} \mathrm{min}^{-1} ; \mathrm{PDI}=1.33$

$2.4 \mathrm{ml} \mathrm{min}^{-1} ; \mathrm{PDI}=1.45$

$2.0 \mathrm{ml} \mathrm{min}^{-1} ; \mathrm{PDI}=1.56$

$1.6 \mathrm{ml} \mathrm{min}^{-1} ; \mathrm{PDI}=1.68$

$1.2 \mathrm{ml} \mathrm{min}^{-1} ; \mathrm{PDI}=1.75$

$0.8 \mathrm{ml} \mathrm{min}^{-1} ; \mathrm{PDI}=1.83$

$0.4 \mathrm{ml} \mathrm{min}^{-1} ; \mathrm{PDI}=1.95$

$0.3 \mathrm{ml} \mathrm{min}^{-1} ; \mathrm{PDI}=2.21$

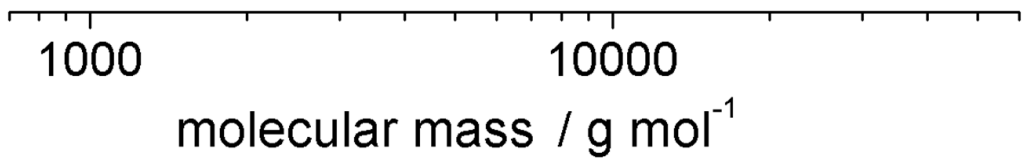

Figure S3: SEC traces for all PS-H-01 $\left(\mathrm{M}_{\mathrm{n}}=3400 \mathrm{~g} \mathrm{~mol}^{-1}\right)$ samples from one reactor setup. (THF, RI signal, PS standards). 


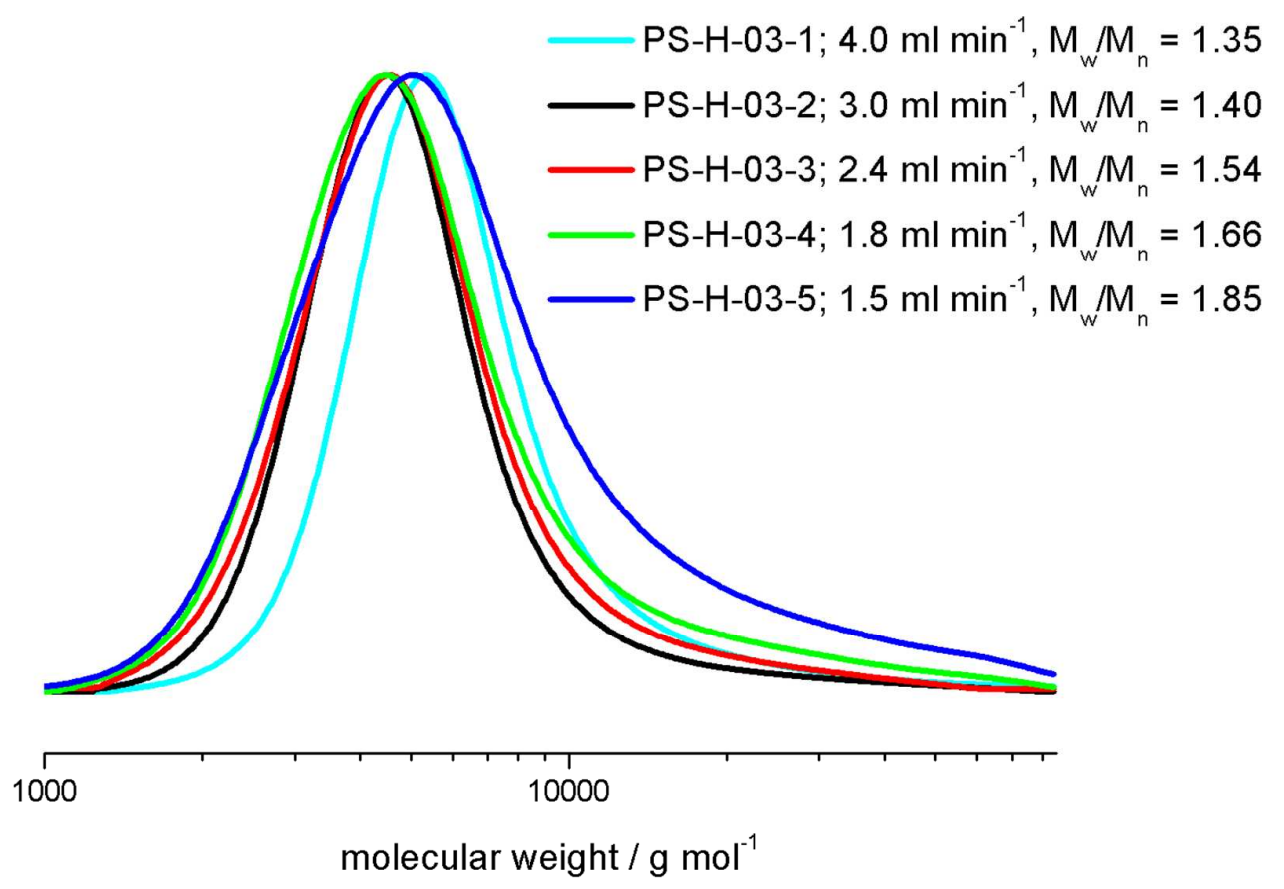

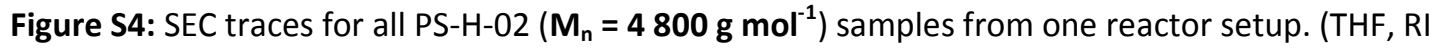
signal, PS standards).

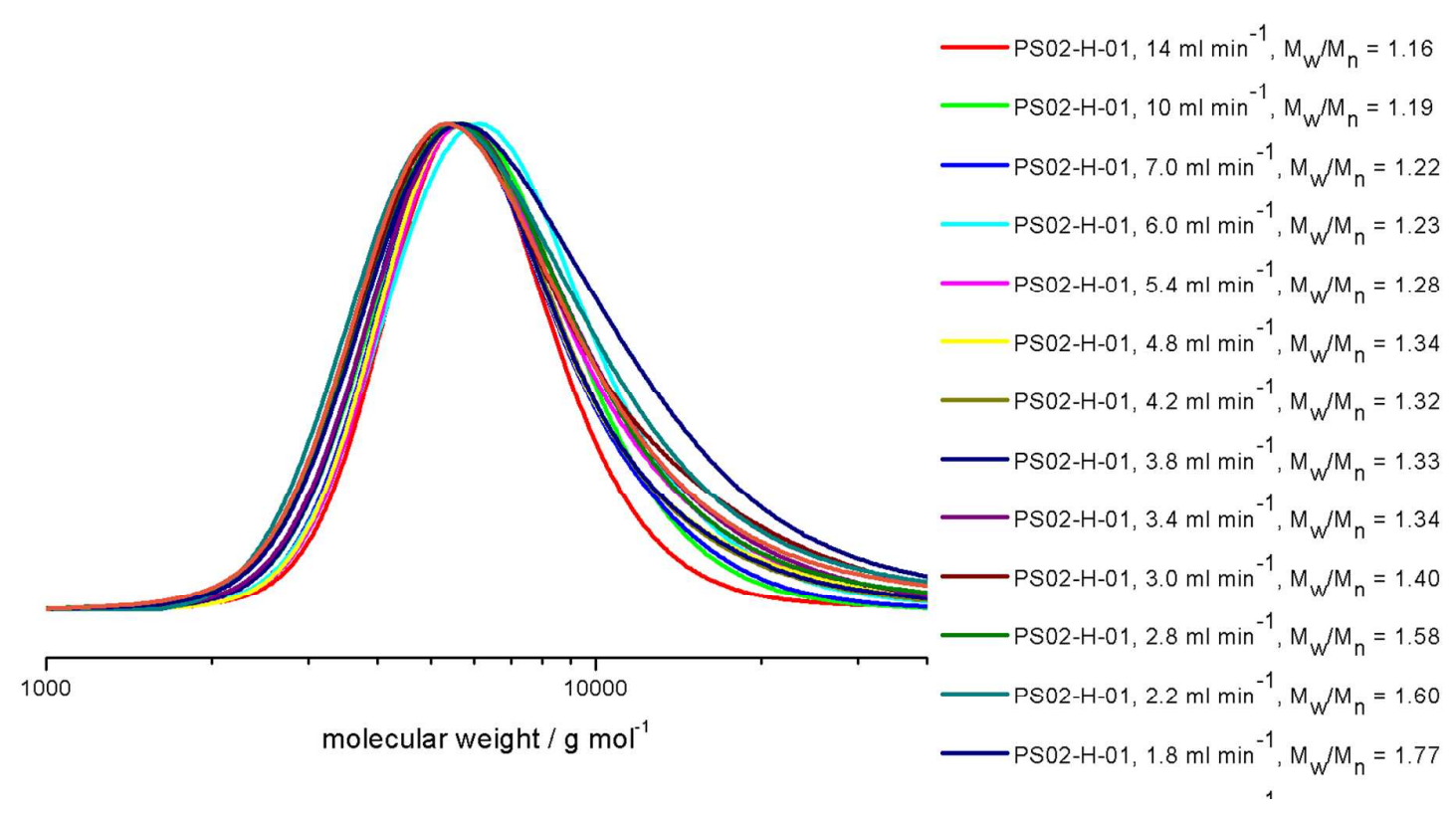

Figure S5: SEC traces for all PS-H-03 $\left(\mathbf{M}_{\mathbf{n}}=\mathbf{5} \mathbf{6 0 0} \mathbf{g ~ m o l}^{-1}\right)$ samples from one reactor setup. (THF, RI signal, PS standards). 


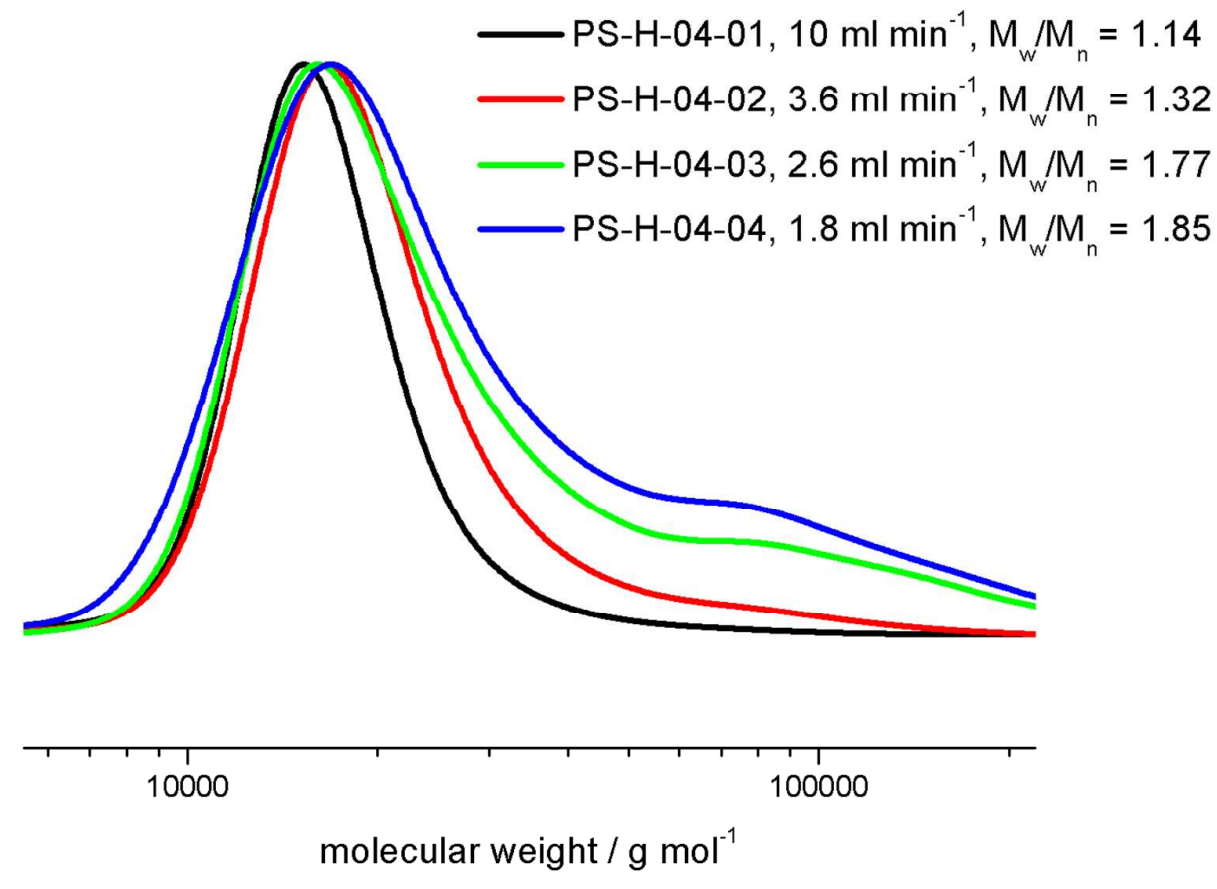

Figure S6: SEC traces for all PS-H-04 $\left(\mathbf{M}_{\mathbf{n}}=18500 \mathrm{~g} \mathrm{~mol}^{-1}\right)$ samples from one reactor setup. (THF, RI signal, PS standards).

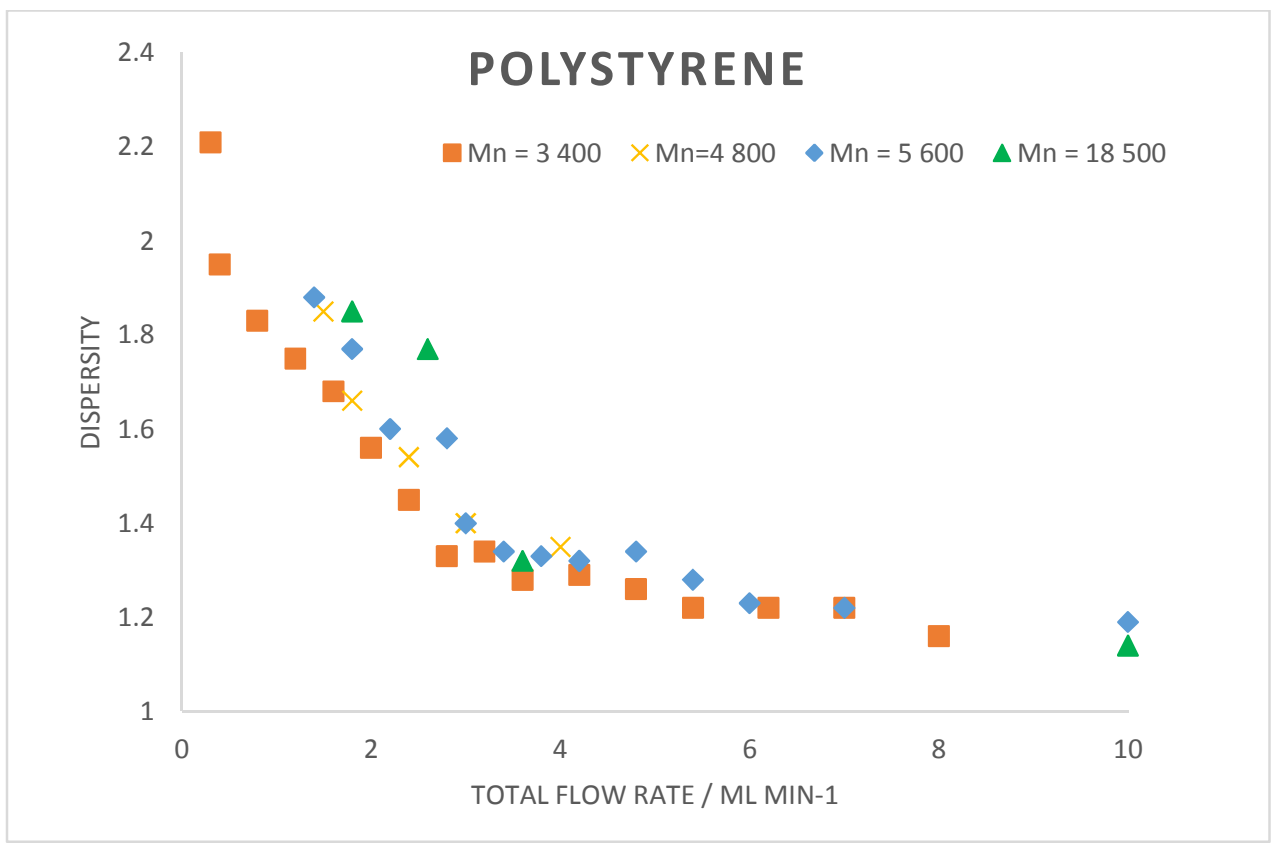

Figure S7: Dispersity vs. total flow rate for four reaction setups with PS with different molecular weights (PS-H-01, $M_{n}=3400 \mathrm{~g} \mathrm{~mol}^{-1}$; PS-H-02, $M_{n}=4800 \mathrm{~g} \mathrm{~mol}^{-1}$ PS-H-03, $M_{n}=5600 \mathrm{~g} \mathrm{~mol}^{-1}$ PS-H$\left.04, \mathrm{M}_{\mathrm{n}}=18500 \mathrm{~g} \mathrm{~mol}^{-1}\right)$. Values obtained by SEC in THF with PS standards. 


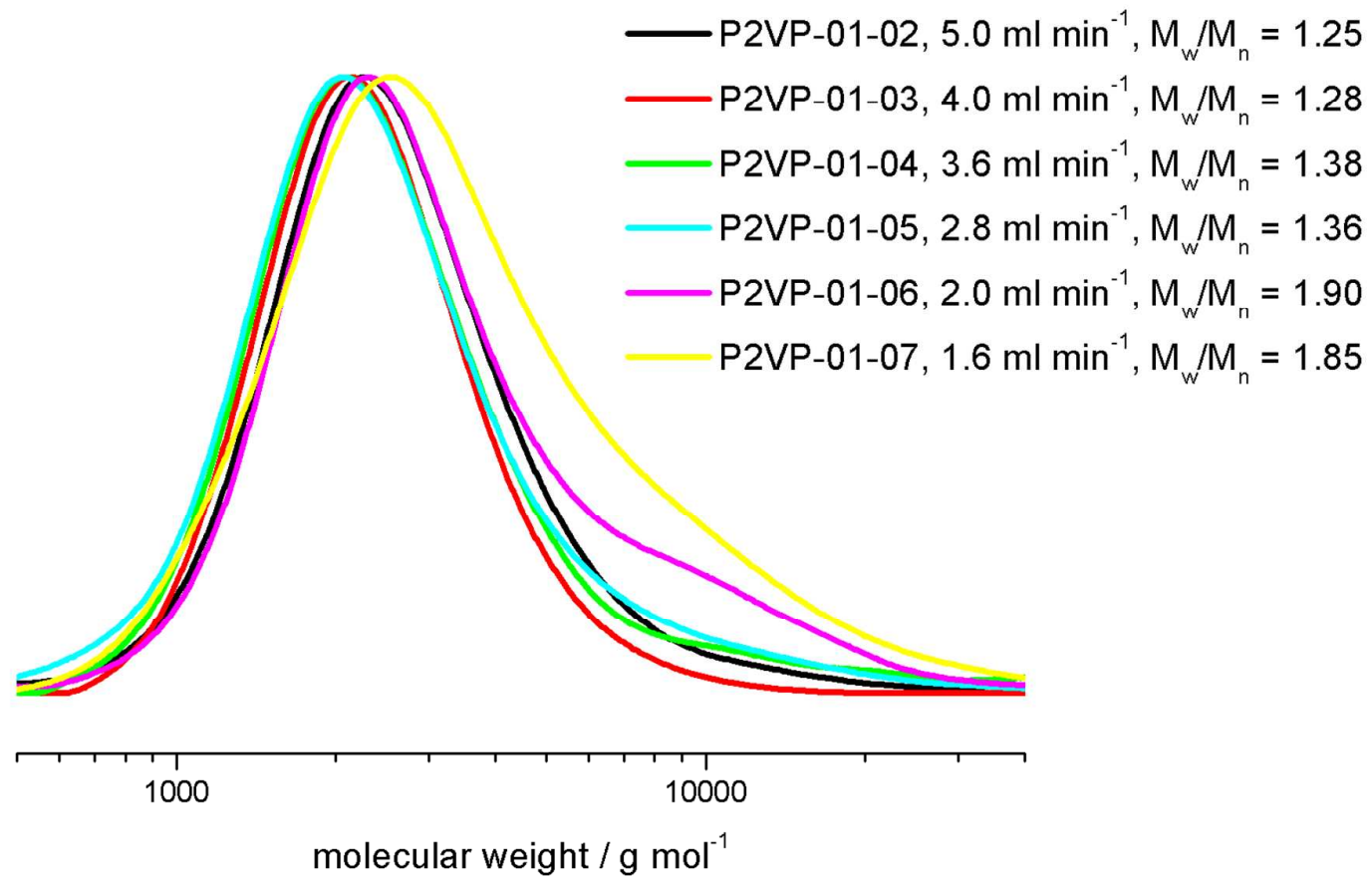

Figure S8: SEC traces for a selection of P2VP samples $\left(M_{n}=2200 \mathrm{~g} \mathrm{~mol}^{-1}\right)$ from one reactor setup. (DMF, RI signal, PS standards).

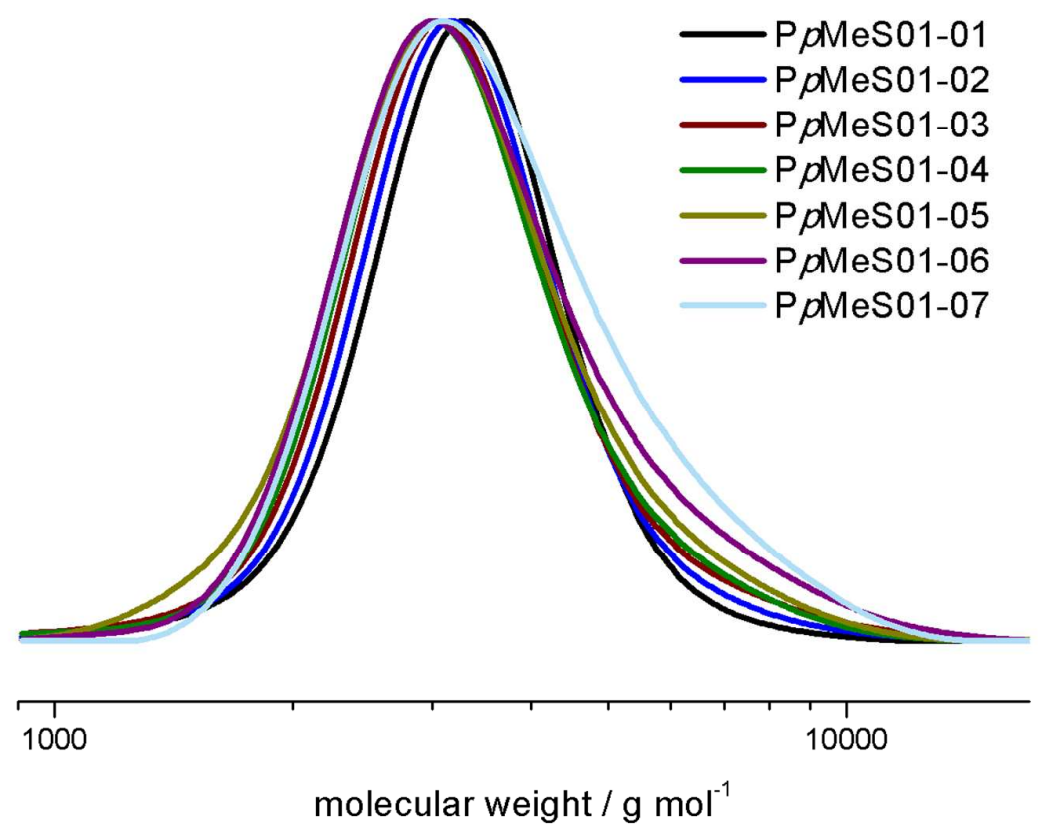

Figure S9: SEC traces for all PpMeS samples $\left(\mathbf{M}_{\mathbf{n}}=\mathbf{3} 100 \mathrm{~g} \mathrm{~mol}^{-1}\right.$ ) from one reactor setup. (THF, RI signal, PS standards). 


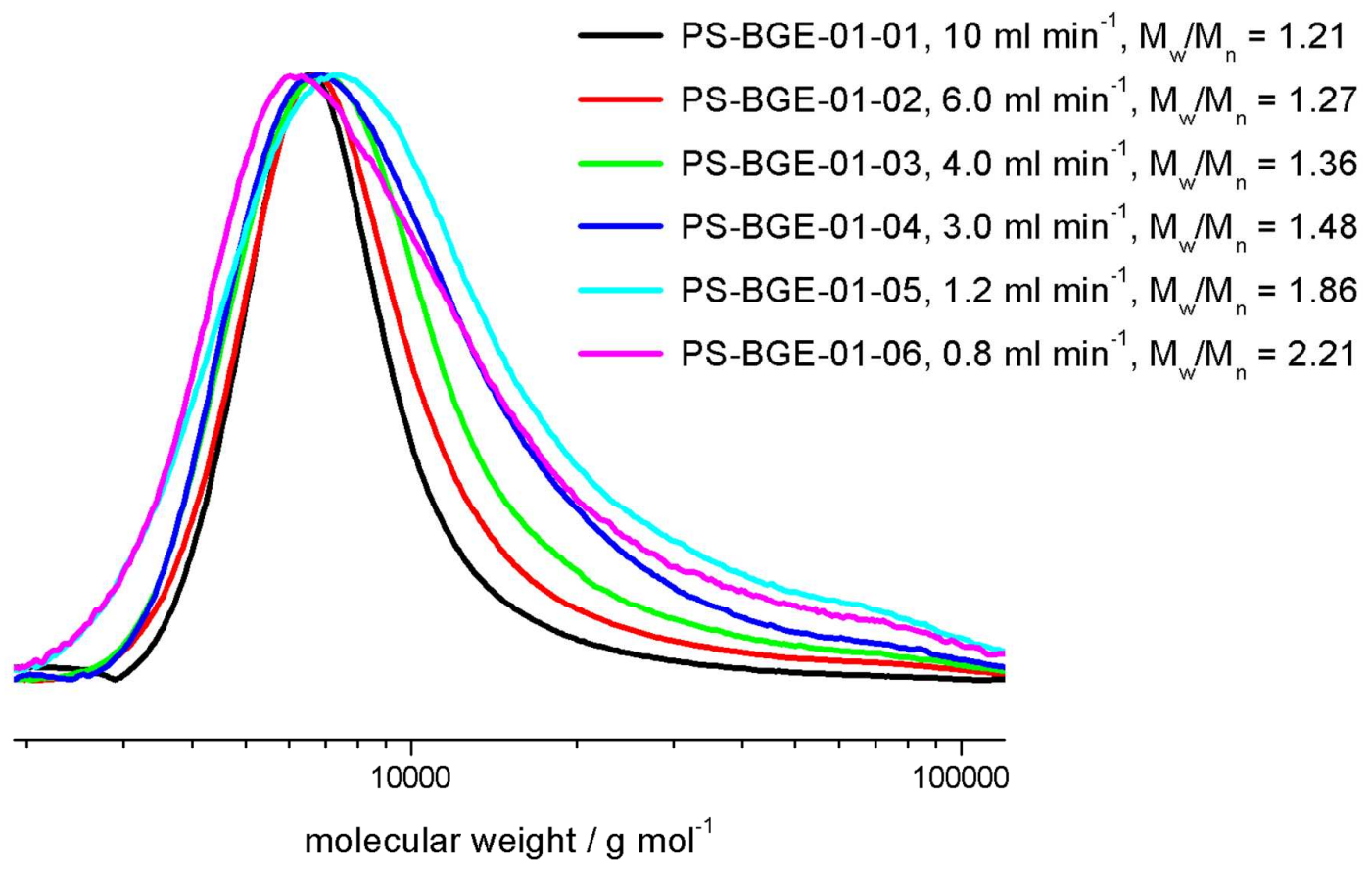

Figure S10: SEC traces for all PS-BGE samples $\left(\mathrm{M}_{\mathrm{n}}=\mathbf{6} 800 \mathrm{~g} \mathrm{~mol}^{-1}\right)$ from one reactor setup. (THF, RI signal, PS standards).

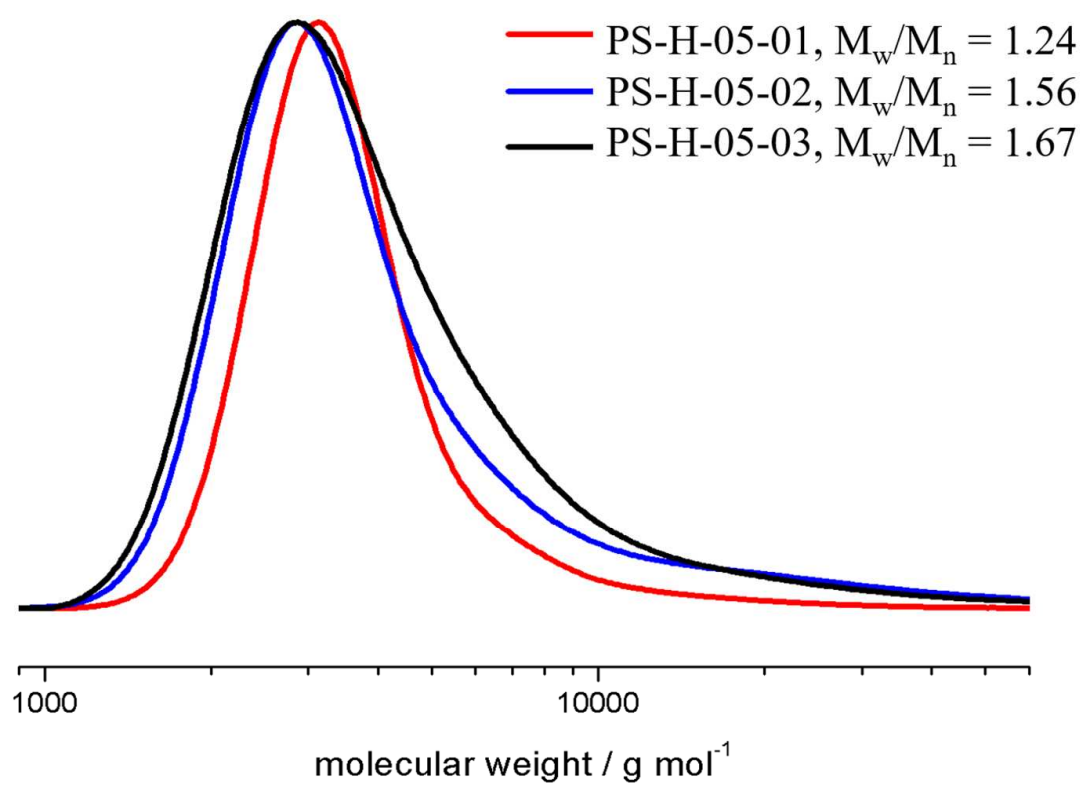

Figure S11: SEC traces for all PS-H-1 samples from one reactor setup. (THF, RI signal, PS standards). 


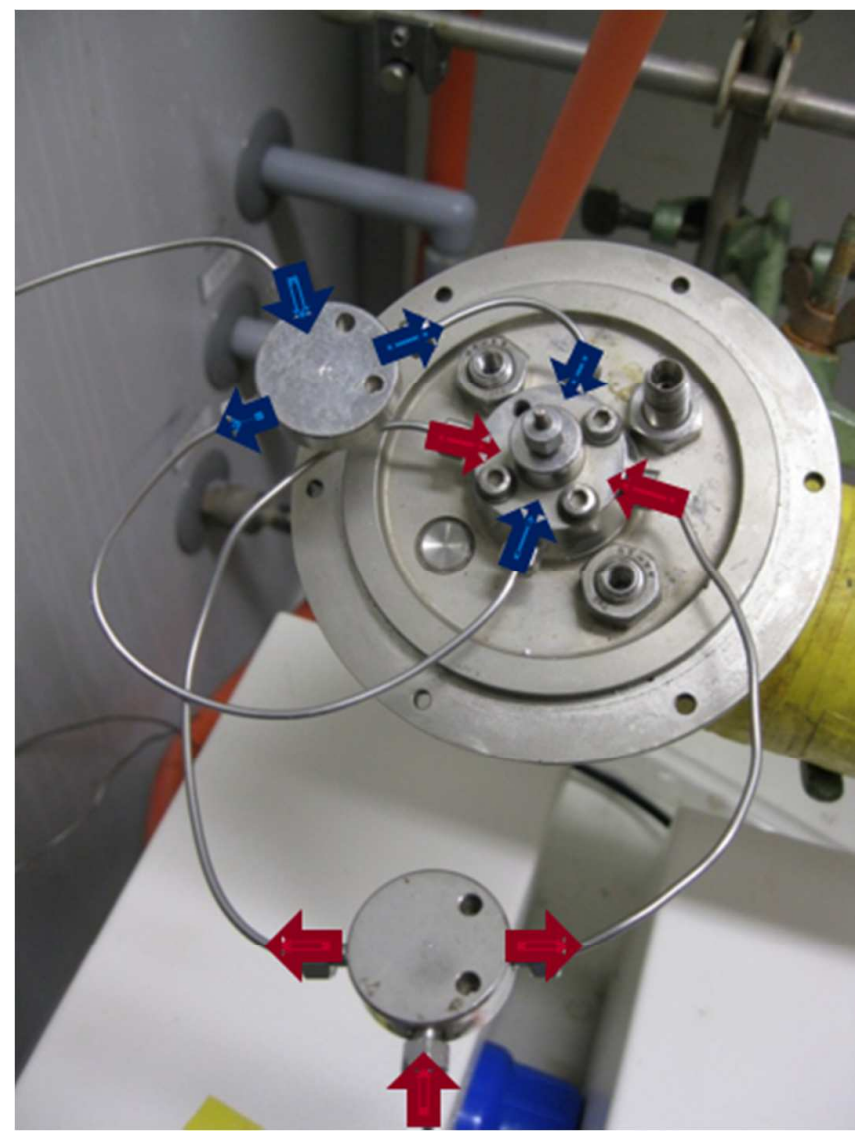

Figure S12: Image of the 4-way jet mixing device with two T-mixers to separate the monomer (blue arrows) and initiator (red arrows) solutions into two streams. 
Table S1. Characteristics of polystyrene samples prepared with different flow rates

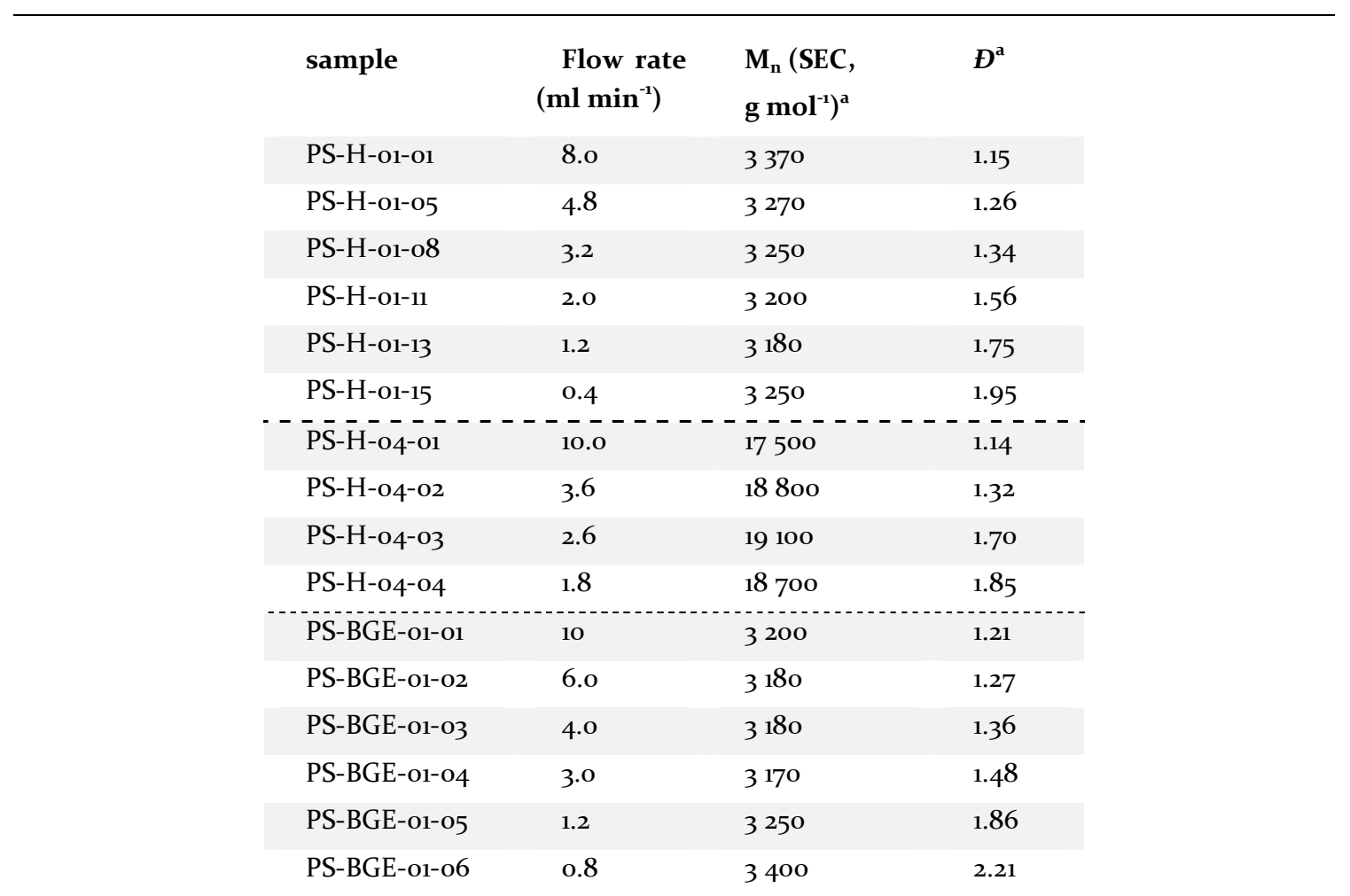

PS-H-01: $[\mathrm{M}]_{0}=1.0 \mathrm{M},[\mathrm{I}]_{0}=0.033 \mathrm{M}$; PS-H-04: $[\mathrm{M}]_{0}=1.0 \mathrm{M},[\mathrm{I}]_{0}=0.006 \mathrm{M}$; PS-BGE-01: $[\mathrm{M}]_{0}=1.0 \mathrm{M},[\mathrm{I}]_{0}$ $=0.020 \mathrm{M},[\mathrm{BGE}]_{0}=0.47 \mathrm{M} ;{ }^{\mathrm{a}}$ Number-average molecular weight and dispersity determined by SEC in THF (PS standards). 


\section{Table S2. Characteristics of PpMeS-H and P2VP samples}

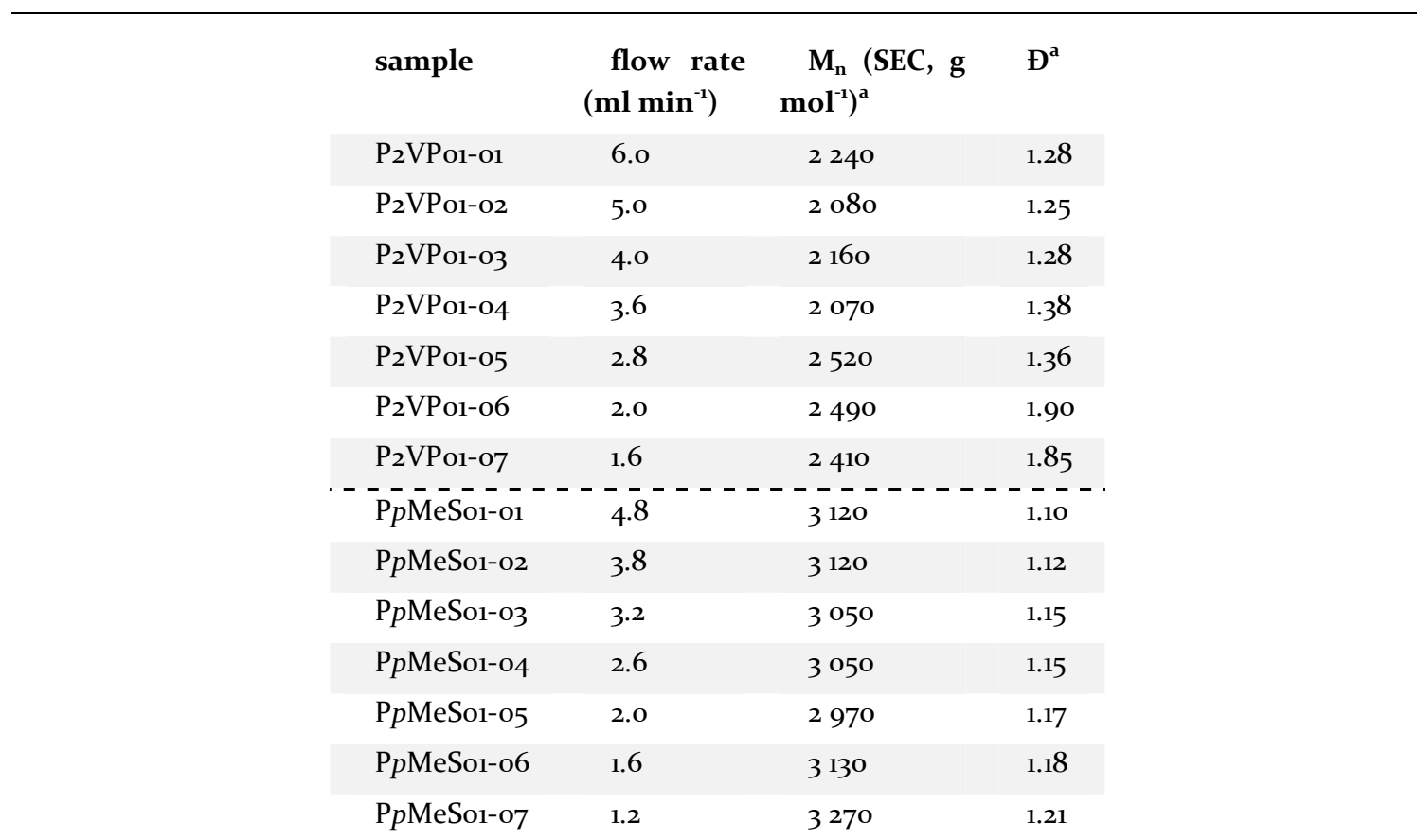

P2VP01: $[\mathrm{M}]_{0}=0.74 \mathrm{M},[\mathrm{I}]_{0}=0.026 \mathrm{M} ; \mathrm{P} p \operatorname{MeS} 01:[\mathrm{M}]_{0}=1.0 \mathrm{M},[\mathrm{I}]_{0}=0.036 \mathrm{M}^{\mathrm{a}}$ Molecular weight determined by SEC in $\mathrm{g} \mathrm{mol}^{-1}$ and molecular weight distribution (PpMeS: PS standards, THF; P2VP; PS standards, DMF). 